\title{
POLITICAS LOCALES DE JUVENTUD: UNA MIRADA AL FONDO DEL OJO
}

\author{
ANDREA IGLESIS LARROQUETTE*
}

\section{A MODO DE INTRODUCCIÓN... QUE ES SIEMPRE UN PUNTO DE PARTIDA}

AMPLIAR LA MIRADA PARA centrar la discusión en los aspectos articuladores de una posible Política Local de Juventud, requiere al menos tres consideraciones básicas. La primera dice relación con la comprensión general de las políticas sociales en la escena nacional, sus tensiones y tendencias; la segunda, con la idea de lo local como concepto emergente y dinámico, y por último, la necesidad de construcción de un mapa general que dé cuenta de la trama o el conjunto de articulaciones entre los diversos niveles de análisis posibles.

De esta forma urge entonces, situarse desde la raíz, desde el gesto de contemplación umbilical, desde el modo del abanico, su dinámica y arquitectura, en otras palabras desde la instalación de una «mirada sospechosa». Esto último no como un imperativo de modè, sino más bien, a propósito de una necesidad política de re-construcción de saberes.

Esto último principalmente a partir del convencimiento de que los grandes avances en materia de juventud pasan necesariamente por

Egresada Psicología Universidad de Valparaíso. Encargada Área de Proyectos y Desarrollo Local, Departamento de Jóvenes, I. Municipalidad de Concepción. 
un cambio de mirada, y con ello de discurso, en relación a los y las jóvenes; en otras palabras, y haciendo aquí una apuesta, de lo que se trata es de renovar las premisas y conceptos desde los cuales se aborda, se construye y actualiza, la relación con el mundo juvenil, venciendo los miedos de enfrentarse desde una perspectiva de sujetos concretos a las realidades del mundo juvenil.

\section{Políticas SOCIALES Y JUVENTUd}

Así, como primer elemento cabe destacar que la política social es un fenómeno específicamente moderno, en tanto que se trata de una acción racional del Estado tendiente a compensar a los sectores sociales desfavorecidos por el mercado, asegurándoles la satisfacción de ciertas necesidades básicas elementales. Por otra parte, las formulaciones de políticas y el modo general en que los problemas sociales son atendidos por el Estado, responden en gran medida al marco conceptual e ideológico en que los gobiernos se desenvuelven, observándose dos niveles decisionales, uno eminentemente político, en el cual se plantean los objetivos referidos a la esfera del poder y del orden social a los que pueden contribuir las políticas sociales, y el otro, asociado a cuestiones técnicas, de efectividad y alcance de las políticas el cual involucra consideraciones de carácter económico de costobeneficio. ${ }^{1}$

Si damos un vistazo general, el interés del Estado por los jóvenes desde los tiempos más remotos, reside en que ellos son el elemento poblacional sustantivo para las finalidades del reclutamiento militar y el esfuerzo bélico. Hasta fines del siglo pasado, los niños y los jóvenes constituyeron una preocupación estatal debido a las formas extremas de implantación del sistema capitalista y la consecuente súper-explotación de la mano de obra. En un segundo momento, y ya conformadas las bases del Estado de Bienestar, la preocupación central se trasladó hacia la valoración de la igualdad de oportunidades. A mediados del siglo el eje de la preocupación estatal por lo juvenil se traslada a las prestaciones sociales socio-sanitarias y de asistencia pública, adquiriendo mayor relieve aquellos sectores de la población juvenil más desvalidos, por limitaciones físicas, mentales o socioculturales.

1 Javier Martínez y Margarita Palacios (1996): Informe sobre la decencia. Santiago: Ediciones SUR. 
La crisis del Estado de Bienestar coloca el tema de lo juvenil desde el punto de vista de las limitaciones de los sistemas educativos, para dar respuesta a contextos socioeconómicos cambiantes, surgiendo más claramente revueltas juveniles asociadas a violencia urbana y a la situación de pobreza, que se caracterizaron por representar demandas desde sectores políticamente no organizados, situación inédita hasta esa fecha.

En Chile, los primeros esfuerzos dirigidos a la población juvenil han surgido básicamente desde una óptica sectorial. De hecho es partir de los años 60 donde se plantean los primeros atisbos de diseño y formulación de políticas en este ámbito, alrededor de la Oficina de Planificación Nacional (ODEPLAN), continuando durante la dictadura a través de la Secretaría Nacional de la Juventud, la cual básicamente desarrolló acciones funcionales al régimen militar.

Durante la década de los 80 y producto de las agudas crisis recesivas que experimentaron las economías latinoamericanas, el sector juvenil comienza a cobrar relevancia en tanto problema social digno de ser investigado. Aquí el eje de la preocupación estuvo centrado en la capacidad de estos sectores para constituirse en tanto movimientos sociales y generar proposiciones alternativas al orden vigente.

En lo que ha sido la evolución de las políticas sociales de juventud en Chile, resulta importante precisar que es a parir de esta década cuando se realizan esfuerzos más orientados hacia la generación de una Política Integral de Juventud.

Aun cuando desde una perspectiva histórica existe un avance en materia de política social de juventud, esto no es suficiente. Estudios e investigaciones desarrolladas durante estos años han puesto de manifiesto las falencias de dicha política, centrando tales deficiencias en cuatro grandes problemas.

El primero da cuenta de la inadecuación de marcos conceptuales a partir de los cuales se ha definido la juventud y las consecuencias que ha traído en relación a la construcción de problema para las políticas sociales. De esta forma, avanzada la década, es posible advertir que el rótulo de daño social y la consecuente reivindicación a través del pago de la deuda social para con los jóvenes, se ha traducido en una satanización y corporalización del daño en el cuerpo de los y las jóvenes Esto se observa con claridad en la proliferación de las ofertas programáticas hacia los jóvenes tendientes a normalizar conductas a partir de imperativos éticos. 
En otras palabras se puede afirmar que no se ha valorado la subjetividad colectiva de los jóvenes al definir las metas últimas de la política social juvenil. Se ha operado con una concepción de desarrollo que sigue sobrevalorando los aspectos socioeconómicos sin dar la adecuada atención e importancia a las dimensiones de la identidad y la calidad de vida.

Por otra parte destaca la falta de coordinación a nivel operativo y conceptual, tanto entre el conjunto de programas del área juvenil como entre los programas relacionados de otras áreas y también entre los programas del área dirigidos a problemas similares. Quizás esta descoordinación es entendible desde la característica de desvinculación intersectorial de nuestra estructura estatal, por un lado; y por otro, por la carencia de una permanente actividad reflexiva que apunte a elaborar y debatir fundamentos conceptuales y técnicos para los programas.

«Esta descoordinación se da también a nivel vertical entre las instancias generadoras de los programas, como el nivel central ministerial, y las instancias ejecutivas a nivel local, como el municipio». ${ }^{2}$ Esta descoordinación se hace visible en las dificultades para implementar adecuadamente los programas de ejecución local, lo cual redunda en que no siempre se utilizan adecuadamente los recursos técnicos y materiales disponibles tanto a nivel central como local. ${ }^{3}$

Otro aspecto importante es la descoordinación que se da entre los programas gubernamentales y los no gubernamentales. Así, aunque habitualmente se reconoce la importancia de la complementariedad entre estos dos tipos de programas, en la práctica se desarrollan pocas iniciativas de complementación efectiva entre ellos. Lo que ocurre en los programas de droga es notorio, las metodologías y los marcos teóricos de cada sector son muy distintos, aunque posibles de complementar, pero su desvinculación no permite potenciarse a través del intercambio. ${ }^{4}$

Otra falencia se refiere a la limitada cobertura en general y su parcial focalización en un sólo nivel de la intervención: de acuerdo con Alfaro, ${ }^{5}$ los programas tienen coberturas que no son suficientes

2 Asún, Alfaro y Morales (1994).

3 Jaime Alfaro (1994): «Los desafíos psicosociales en la realidad juvenil y la respuesta institucional del Estado». Primer informe nacional de juventud. Santiago: Instituto Nacional de la Juventud.

$4 \quad$ Asún, Alfaro y Morales: Op. cit.

5 Jaime Alfaro: Op. cit. 
para las demandas que se presentan en general, siendo necesario indicar que se ha incrementado significativamente la asignación de recursos sobre todo en el área de la prevención.

Por último, se observa la inexistencia de un sistema de evaluación. Prácticamente no se desarrollan evaluaciones sistemáticas de la eficacia ni de la eficiencia de estas acciones estatales. En algunos casos se cuenta con evaluaciones de tipo descriptivo o de cobertura, pero no se conocen públicamente evaluaciones cuantitativas ni cualitativas rigurosas de sus productos. «Es más, existe una creciente sospecha en algunos programas, que la llegada de ellos tiende a ser en los grupos de riesgo intermedio y no en los grupos de alto riesgo, originales destinatarios». 6

De esta forma observamos que aun cuando las cifras generales de promedios pueden indicarnos que la pobreza juvenil efectivamente ha disminuido, las diferencias que existen entre los jóvenes son más abismantes que en cualquier otro caso, profundizándose las diferencias de género, clase, territorialidad, etc.

Este panorama multiforme ha contribuido a generar una impresión caleidoscópica en la construcción de acciones dirigidas al mundo juvenil, proveniente más de las dificultades de organizar una acción coherente y de un desconocimiento básico de la construcción de mundo elaborada por las y los jóvenes de los 90 .

Sin embargo, durante los últimos años, y a propósito de una navegación en tempestad sin faro, se han comenzado a desarrollar un ejercicio de análisis y revisión de las prácticas que desde la institucionalidad existente en materia de juventud se han realizado, las cuales han comenzado a puntualizar y dar énfasis en aspectos más vinculados a procesos que apuntarían al fortalecimiento democrático, tarea en la que hasta hoy día sólo se ha avanzado a nivel declarativo.

Este ámbito de acción, todavía difuso, da cuenta de un ejercicio de cambio de lente, en cuanto que implica el comenzar a preguntarse hasta qué punto es posible continuar desarrollando intervenciones orientadas a propiciar la igualdad de oportunidades sin abordar de fondo el tema del desarrollo social. 


\section{LAS POLÍTICAS SOCIALES DE JUVENTUD EN EL MARCO DEL DESARROLLO LOCAL}

Hacer referencia a lo local remite a un sinnúmero de proposiciones que van desde reformas del Estado, a nociones sobre la democracia, pasando por concepciones de política regional, tipos de organización social, a la defensa o construcción de identidades y autonomías, a modalidades de política social, al reconocimiento de sujetos empresariales y al modo de apoyarlos y la preocupación por el respeto de la biodiversidad.

Desde una lectura crítica este concepto se expresa, respectivamente como burocracia, ineficiencia e ilegitimidad estatales, déficit democráticos en la orientación de la sociedad, insuficiente vitalidad de la sociedad civil, límites del desarrollo regional, homogeneización cultural, ineficiencias e inequidades de la política social, inexistencia de suficientes trabajos y empleos, una sociabilidad empobrecida, crisis sectoriales en el ámbito de la acción pública, deterioros ambientales amenazantes para un desarrollo sustentable.

Sin embargo, a pesar de la aparente dispersión del concepto, es importante constatar que en todos estos temas, los espacios locales son considerados relevantes para enfrentar situaciones que se definen como indeseables, y como componentes significativos de un cambio de realidad.

Aunque buena parte de estas valorizaciones de lo local, más que ser propuestas de desarrollo local, en tanto entenderemos por éste algo que abarca de manera totalizadora e integrada los componentes de una realidad y de su desarrollo, han constituido valoraciones parciales referidas a algunas de las múltiples problemáticas ya descritas. Más allá de lo puramente declarativo, estas valorizaciones han fundamentado o dado un sentido renovado a iniciativas o transformaciones que han alterado, en grados diversos de importancia, las realidades locales. Aquí me refiero a traspasos de recursos y competencias a los municipios, a la focalización de programas públicos, a la animación de grupos locales con capacidad de acción frente a determinadas carencias, al rescate de identidades locales que harían parte de la identidad local, a políticas de promoción empresarial, a la sensibilización sobre el patrimonio natural local, a la generación de grupos de presión para obtener determinadas políticas regionales y municipales, entre otras.

De esta forma, aunque buena parte de estas valorizaciones, propuestas y acciones referidas a lo local, dado su carácter parcial o 
específico, no logran establecer una relación de contenido particular con una mirada amplia sobre las dimensiones presentes en la realidad local y, consecuentemente, con lo que constituiría una propuesta integral de desarrollo local, no se puede desconocer la incidencia que éstas tienen en el desarrollo de la localidad.

Sin embargo, el qué es, para qué sirve, quiénes lo pueden construir y cómo, son preguntas de actualidad respecto del desarrollo local que reclaman construir un campo más explícito y transparente de debate, razón por la cual se hace necesario un marco referencial.

Raúl González Mayer, propone una marco referencial constituido por cuatro ejes. El primero lo denomina el eje de los contenidos o dimensiones del desarrollo local. En este terreno las dimensiones esenciales se refieren a lo cultural-identitario, a lo político, a lo económico y a lo público-administrativo. En cada una de estas dimensiones la localidad puede ganar o perder peso, y con ello, encontrarse en trayectos ascendentes o recesivos de desarrollo local.

Un segundo eje que debe estar presente en la elaboración de una propuesta de desarrollo local se refiere a las escalas que deben ser consideradas. Esto significa que deben entenderse referidos a espacios socio territoriales que comprenden escalas barriales, comunales, micro regionales y regionales. Esto requiere a su vez, el fortalecimiento de unidades territoriales de acción que expresen procesos sociales, situaciones comunes e identificaciones ya existentes. Por otra parte necesita de una articulación estratégica entre las distintas escalas.

El tercer eje dice relación con los agentes, que en un sentido amplio, pueden ser los animadores de procesos de este tipo. En este sentido se afirma que el impulso debe ser intencionado tanto por agentes sociales como estatales. Esto quiere decir que se supone tanto un proceso de descentralización del Estado como de fortalecimiento de la sociedad civil local, presuponiendo además una mutua interdependencia.

Por último, un cuarto eje constitutivo se refiere al marco de interdependencia mundial actual. En este sentido, una propuesta de desarrollo local supone la constitución de un importante poder local que pueda expresar, realizar y defender lo que se vaya constituyendo de manera puntual o estratégica como un interés local.

He hecho este largo recorrido para argumentar y connotar la importancia de instalar los procesos de descentralización del Estado y lo que hemos llamado la dimensión pública-administrativa en el marco de un proceso de desarrollo local complejo. Aunque resulte trivial 
decirlo, lo local no es sinónimo únicamente de descentralización estatal o de la acción municipal. En este sentido el avance que debe delinearse en cuanto a los procesos de construcción de Políticas Locales de Juventud no sólo debe estar aparejado a la consolidación de equipos técnicos al interior del municipio y/u otras instancias estatales o al traspaso de recursos desde el nivel central.

Debe trabajarse además, y en especial durante la primera etapa, el resto de los ejes antes descritos, esto significa, principalmente instalar plataformas de acción que impliquen el relevamiento y fortalecimiento del actor juvenil desde su propia lógica particular, así como el generar instancias de articulación entre los múltiples niveles de la realidad local.

Otro tema que surge con fuerza tiene que ver con el ejercicio compartido de las cuotas de poder, situación en que aparecen francamente en desmedro las y los jóvenes producto de la visión estigmatizada y la construcción de sujeto joven elaborada por las políticas públicas, además de los dispositivos de sospecha e intolerancia que operan contra los y las jóvenes.

\section{UNIDADES MUNICIPALES SE JUVENTUD: UNA MIRADA AL FONDO DEL OJO}

Pongámonos de lado de los municipios. Haciendo un rápido recorrido por las Unidades Municipales de Juventud (UMJ), la instancia llamada a generar políticas de juventud a nivel local, es fácil advertir que el panorama es muy diverso. Estructura, funciones, recursos humanos, financiamiento, son variables que permiten pasearse desde un área de jóvenes a cargo de una persona que además está encargado/a de la tercera edad, la infancia y las mujeres y que cuenta con un millón de pesos para desarrollar el trabajo del año (o menos) hasta un departamento de jóvenes con un equipo estable de profesionales con dedicación exclusiva al tema y con recursos financieros de varios millones más.

Debo decir que aunque resulte más o menos claro determinar óptimos en cada una de estas variables, el tema no se resuelve ahí. O en otras palabras, y haciendo un ejercicio de imaginería, aunque todas las Unidades Municipales de Juventud contaran con un reconocimiento expresado en una estructura interna de alto rango (lo que de una manera u otra expresaría la relevancia política del tema), tuvieran equipos de profesionales implicados permanentemente en el trabajo 
con jóvenes (o sea la validación de la figura de un/a experto/a en juventud) y contaran con los recursos suficientes y necesarios (aunque éste sea un concepto poco adecuado al espíritu neoliberal que nos inunda), aún quedarían nudos bastante importantes que resolver, los cuales están muy asociados a la construcción de lo juvenil en estos tiempos.

Sin embargo y haciendo una abstracción muy rápida es posible afirmar que son tres los grandes ámbitos de trabajo que se realizan desde las UMJ:

- Subvención de la demanda juvenil de la comuna (los/as que pueden acceder a esa demanda principalmente- «los»).

- Realización de eventos masivos que convocan a la juventud.

- Labor de contención de "problemas psicosociales», básicamente a través de la prevención.

Todas estas áreas de trabajo centran principalmente su accionar en sujetos colectivos (organizaciones juveniles, centros de alumnos, bandas de rock, otros). Este acercamiento a la cotidianeidad juvenil permite ir construyendo relaciones más naturales con una cierta franja de la población juvenil, que no siempre es la más marginada; generando una relación básicamente con hombres jóvenes, en la medida que son los hombres los líderes de las organizaciones (debido principalmente a la subordinación de la mujer en las culturas patriarcales como la nuestra), y a los cuales se les ofrecen de preferencia alternativas de uso de tiempo libre (o vacío).

Todo esto se traduce en la implementación de intervenciones con los grupos pobres «más integrados», desde la óptica de los colectivos, lo cual determina una cierta mirada particular hacia él o la joven interlocutor/a en la medida que es siempre representante de, y principalmente con hombres jóvenes.

Claro está que este acercamiento es el punto de partida real que tienen las UMJ y resulta vital asumirlo y comprenderlo para desde allí generar estrategias de intervención más coherentes y centradas. De una u otra forma no tener conciencia de cómo se está mirando a la juventud o cómo te permite mirar la juventud la estructura municipal, para mi gusto, es una de las falencias más graves de las UMJ. 
Desde esta mirada hacia la juventud que permite el municipio, (la que resulta ser la imagen estereoscópica de una débil y desarticulada política de juventud, la poca relevancia política del tema y la burocracia municipal que ordena la relación con la comunidad de ocho treinta a cinco treinta de la tarde y no valora los esfuerzos profesionales ni económica ni moralmente de sus funcionarios/as), es posible percibir al menos tres grandes nudos presentes en la dinámica de las Unidades Municipales de Juventud.

\section{a) El «eventismo» o la tentación de la parafernalia}

Existe la tendencia de evaluar la gestión de las UMJ en base al impacto de los eventos que realiza, la cantidad de gente que moviliza en torno a shows masivos con rótulo juvenil, la calidad o el renombre de sus protagonistas u otros parámetros propios de un estudio de marketing.

De una u otra forma la gestión desde el municipio se debate entre la figura de una productora de eventos y una estructura de normalización de conductas «desviadas» o poco adecuadas (desde aquí resulta impensable no trabajar el tema del consumo de drogas, SIDA, embarazo adolescente, entre otros).

Este fenómeno no es propio solamente de la gestión institucional. De una u otra forma el megaevento es el ícono de la cultura light en que vivimos, el momento mágico casi al alcance de la mano.

Sin embargo y de nuevo insisto. No es en sí mismo éste el problema.

El problema surge en la medida que la UMJ se transforma en productora, en la medida que el objetivo se transforma en la actividad puntual, cuando restringe el espacio y acorta la dimensión de proceso, de ensayo y error, de maduración, de ruptura, de negociación y encuentro, cuando no transfiere y comparte experticias con los y las jóvenes, cuando invisibiliza a las jóvenes, cuando olvida la riqueza del transcurrir.

Hay en este tipo de actividades un enorme desafío, el de ceder el micrófono en las conferencias de prensa, el permitir que emerjan las voces de los y las jóvenes, en relevar procesos de actoría que se van consolidando.

Pero como una sombra persiste la tentación de la parafernalia. 


\section{b) La esquizofrenia de la Unidad Municipal de Juventud}

Algunas de las paradojas esquizofrénicas de la dinámica municipal son de conocimiento público. Quizás las de las UMJ son igualmente predecibles.

La UMJ debe responder de acuerdo a los ritmos de la dinámica juvenil con la capacidad de respuesta de la municipalidad, por lo tanto tiene que hacer magia o conformarse a trabajar con los conformados.

Los municipios optan por contratar jóvenes para intervenir con jóvenes, pero no considera sus opiniones ni propuestas porque son jóvenes.

Así podríamos nombrar unas cuantas más, se los dejo como ejercicio, pero lo importante aquí resulta ser otra paradoja. Cuando un interventor en juventud tiene la destreza suficiente para manejarse con soltura en estos ámbitos, ya está muy viejo para trabajar en juventud (según los parámetros de sentido común la gente piensa que se trabaja en juventud mientras se es joven o hasta que encuentre algo mejor - cosa que ocurre cuando se deja de ser joven-, no en vano juvenólogos de vasta experiencia no abundan en nuestro país).

\section{c) ¿La primera o la última pieza del rompecabezas?}

Dentro del la estructuración jerárquica de la Política de Juventud se tiende a afirmar que el trabajo que desempeñan las Unidades de Juventud constituye la primera línea de intervención con los y las jóvenes. Ésta se desarrolla inserta en el espacio local y por lo tanto adquiere o debería adquirir insumos de primera mano para desarrollar propuestas atingentes a las demandas de desarrollo social juvenil de la comuna.

Por otra parte las Unidades de Juventud desarrollan acciones que otras instancias o programas orientadas hacia la juventud no realizan, ni a nivel local ni nacional. De esta forma las políticas de salud, educación, capacitación, empleo, fomento productivo, entre otras dirigidas a los/as jóvenes, no pasan por la UMJ. Así como las acciones de las UMJ no se relacionan con éstas.

Aquí entonces se evidencia claramente la falencia en la coordinación de las políticas de juventud, lo que va tensionando el sistema en la medida que es impensable e insostenible la generación de políticas locales sin niveles de coordinación sustantivos. 


\section{UN MODELO PARA ARMAR}

Sin el ánimo de dar por cerrada la discusión, sino precisamente con el objeto de aportar nuevos elementos que permitan ir profundizando la reflexión y avanzando hacia la construcción de una Política Local de Juventud participativa o desde los jóvenes, es que en esta parte intentaremos desarrollar una línea prospectiva en relación a lo que deberían ser los objetivos estratégicos de las Unidades Municipales de Juventud.

En líneas generales podríamos afirmar que se deberían trabajar - al menos a juicio nuestro- dos grandes tipos de objetivos, que podríamos denominar de primer y segundo orden.

\section{a) Objetivos de primer orden}

- Constituirse en la primera línea de intervención con los y las jóvenes de la comuna. Objetivo que podría lograrse a través de la construcción de una relación permanente con los y las jóvenes apostando a una presencia constante de animadores socioculturales en los diversos sectores, respetando la dinámica del mundo juvenil (horarios, espacios de encuentro, territorialidad, género, emergencias temáticas, entre otros), apostando de esta forma a la construcción de una relación no sólo vinculada a la demanda puntual y no sólo con una entrada desde lo colectivo juvenil.

- Consolidar procesos de actoría social y construcción de ciudadanía juvenil, brindando las herramientas necesarias para facilitar dichos procesos.

- Aportar elementos críticos que permitan reflexionar en torno a elementos de construcción cultural del espacio local (género, clase, territorialidad, entre otros).

- Propiciar la igualdad de oportunidades entre los géneros. Lo que se debería traducir en una intervención directa con mujeres jóvenes, al menos en una primera instancia.

\section{b) Objetivos de segundo orden}

Éstos básicamente dicen relación con los esfuerzos de dar coherencia a las intervenciones en juventud en la localidad. 
- L La UMJ como enlace facilitador en la construcción de sentidos.

- Coordinar coordinaciones a nivel local y desde el nivel local.

- $\quad$ Posicionar política y públicamente los temas y las tensiones emergentes desde el mundo juvenil.

- Validar la UMJ como organismo técnico al interior del municipio y en la localidad.

Claro está que asumir estos objetivos implica un trabajo de creatividad, experticia en relación al trabajo con jóvenes y dominio institucional, sin embargo aquí se encuentra el desafío, el de un modelo por armar.

CONCEPCIÓN, FEBRERO DEL 2000 